advantages in supplementing the photographic records by direct eye-observations. I regret that the very few occasions on which it has been possible to observe the sun has put it out of my power to make further experiments in these and some other obvious directions.

P.S. - [I have Capt. Abney's permission to add the following letter this day received from him ;-" A careful examination of your series of sun-photographs, taken with absorbing media, convinces me that your claim to having secured photographs of the corona with an uneclipsed sun is fully established. A comparison of your photographs with those obtained during the eclipse which took place "in May last, shows not only that the general features are the same, but also that details, such as rifts and streamers, have the same position and form. If in your case, the coronal appearances be due to instrumental causes, I take it that the eclipse photographs are equally untrustworthy, and that my lens and your reflector have the same optical defects. I think that evidence by means of photography of the existence of a corona at all is as clearly shown in the one case as in the other."December I 5, I882.]

\section{A WEDGE AND DIAPHRAGM PHOTOMETER}

A NEW photometer, shown in perspective in the figure, A has lately been constructed by Mr. Sabine. The stand supports a straight horizontal tube, at one end of which is a paraffin lamp, and at the other an eyepiece. The middle portion of the tube is cut away, and has, slipped over it, a collar to which a frame is attached, carrying a wedge of neutral-tinted glass, adjustable by means of a rack and pinion. Inside the collar is fixed a transverse disc of ground opal glass, which the paraffin lamp illuminates to a definite degree. This disc constitutes the field of comparison, the illumination of which is

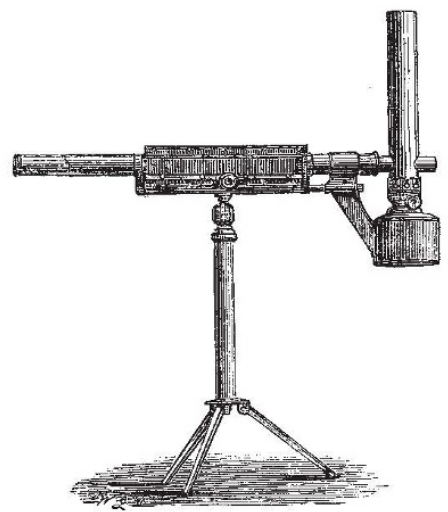

adjustable by means of a series of diaphragms of known aperture at the end near to the paraffin lamp. At the side, between the wedge and the collar which carries it, is a narrow pane of ground opal glass, just behind which a small mirror is fixed at an angle of $45^{\circ}$ to the axis of the tube. This mirror is supported from the centre of the transverse opal disc in such a way, that the support is hidden from the observer by the mirror itself, an arrangement which insures the apparent juxtaposition of the illuminated surfaces which have to be compared. The light to be measured is placed on the right-hand side of the photometer; and the collar is turned so that the light falls normally upon the face of the wedge, passes through the wedge, through the pane of opal glass, and is incident upon the mirror, which reflects a portion of it to the eye of the observer. The wedge is then shifted, if necessary, to interpose a greater or less thickness of absorbing medium, until a balance is obtained, that is until the apparent illumination of the mirror is equal to that of the field of comparison, in the midile of which it is seen. If the range of the wedge is insufficient to admit of this, the degree of illumination of the field is altered, by means of the diaphragms, and the wedge is then adjusted.

The employment of glass wedges for photometric comparisons is not new, having been already used by both Xavier de Maistre and Quetelet; but no practical photometer based upon this method has hitherto been constructed. The employment of diaphragms for extending the range of the wedge is found to work well and to enable the operator to adjust the illumination of the field with exactitude, the bright part of the paraffin flame being of course, kept opposite to, and so as to well cover the diaphragm aperture. A table is constructed giving for each position of the wedge and for each diaphragm, the value, in standard candles, of any light placed at a distance of one metre from the instrument; and if the light be placed at any other distance, the number in the table has simply to be multiplied by the square of the actual distance in metres. For ascertaining approximately the amount of light which passes through any given coloured glass, for example, orange glass, the eyepiece is furnished with a rotary disc containing small panes of white and different coloured glasses, either of which can be interposed at pleasure.

This photometer is being made by Messrs. Elliott Bros., in two forms, one for use as a portable photometer, as shown in the figure, and the other on a more solid stand, for laboratory purposes.

ON THE OCCURRENCE OF GREAT TIDES SINCE THE COMMENCEMENT OF THE GEOLOGICAL EPOCH ${ }^{\mathrm{I}}$

$\mathrm{I} \mathrm{T}$ will I daresay be within the recollection of many of those who are now present that I was honoured by the invitation to deliver the opening lecture in this hall last year. In response to that invitation I addressed to you a discourse which I ventured to call "A Glimpse through the Corridors of Time." Accounts of it have appeared in very many quarters, both at home and abroad. I am myself responsible for the account which appeared in the columns of NATURE, as well as for the pamphlet form in which the lecture has since been issued. The chief reason why I now recur to the subject remains to be stated. Among the various comments which have been made upon that address, some are by no means favourable to the views I ventured to put forward, and they have been the theme of considerable discussion. $\mathrm{Up}$ to the present I have not made any reply to the criticisms which have appeared ; I postponed doing so until a suitable opportunity should have arisen for a review of the whole subject. Your kindness in inviting me once again to address this great Institute has afforded such an opportunity, and with your permission I propose to preface the subject of my lecture this evening by a reply to those critics who have honoured me with their attention.

Let me recall to you very briefly the subject of that lecture, so as to enunciate clearly the point as to which an issue has been raised. You will perhaps recollect that the lecture treated principally of the tidal relations between the earth and the moon, of the influence of the tides during ages past, and of the future which awaits the earth-moon system during ages to come. I pointed out that at the present moment the orbit of the moon must be gradually growing in size, that this gradual increase of the distance from the earth to the moon is essentially non-periodic, and thus is totally different to the ordinary lunar irregularities which are recognisted in rigid-body astronomy. As a consequence of this incessant growth in the moon's distance we see that in past ages the moon must have been appreciably nearer to the earth than it is

I Extract from a lecture delivered at the Midland Institute, Birmi:gham, on November 20, 1882, by Prof. Robert S. Ball, LL.D., F.R.S. Conmunicated by the Author. 